\title{
THERMAL AND CHEMICAL EVOLUTIONS OF GALAXY CLUSTERS OBSERVED WITH SUZAKU
}

\author{
Kosuke SAto $^{a, *}, \mathrm{KyOKO}_{\text {Matsushita }}^{a}$, Yoshitaka Ishisaki $^{b}$, \\ NORIKO Y. YAMASAKI ${ }^{c}$, TAKAYA OHASHI ${ }^{b}$ \\ ${ }^{a}$ Department of Physics, Tokyo University of Science, Japan \\ ${ }^{b}$ Department of Physics, Tokyo Metropolitan University, Japan \\ ${ }^{c}$ Institute of Space and Astronautical Science (ISAS), Japan Aerospace Exploration Agency, Japan \\ * corresponding author: ksato@rs.tus.ac.jp
}

\begin{abstract}
We studied the properties of the intracluster medium (ICM) of galaxy clusters to the outer regions observed with Suzaku. The observed that the temperature dropped by about $\sim 30 \%$ from the central region to the virial radius of the clusters. The derived entropy profile agreed with the expectation from simulations within $r_{500}$, while the entropy profile in $r>r_{500}$ indicated a flatter slope than the simulations. This would suggest that the cluster outskirts were out of hydrostatic equilibrium. As for the metallicity, we studied the metal abundances from $\mathrm{O}$ to $\mathrm{Fe}$ up to $\sim 0.5$ times the virial radius of the galaxy groups and clusters. Comparing the results with supernova nucleosynthesis models, the number ratio of type II to Ia supernovae is estimated to be $\sim 3.5$. We also calculated not only Fe, but also $\mathrm{O}$ and $\mathrm{Mg}$ mass-to-light ratios (MLRs) with K-band luminosity. The MLRs in the clusters had a similar feature.
\end{abstract}

KEYworDS: galaxy clusters, X-rays, thermal evolution, chemical evolution.

\section{INTRODUCTION}

Clusters of galaxies, the largest virialized systems in the universe, are filled with the intracluster medium (ICM), which consists of X-ray emitting hot plasma with a typical temperature of a few times $10^{7} \mathrm{~K}$. X-ray spectroscopy of the ICM can immediately determine its temperature, mass and metal abundances. The mass profile of a cluster, which is a useful parameter for constraining cosmology, is determined through $\mathrm{X}$-ray measurements of the temperature and density structure of the ICM under the assumption of hydrostatic equilibrium of the ICM. In the framework scenario of a hierarchical formation of structures based on the cold dark matter (CDM) paradigm, clusters are also thought to grow into larger systems through mass accretion flows along large-scale filamentary structures. Consequently, the ICM properties to the virial radius play key roles in investigating the structure formation and the evolution of clusters. Because of the difficulties in observation, however, properties, such as temperature, density, pressure, entropy, and metal abundance around the virial radius are not yet known. Also, the metal abundances of ICM provide a large amount of information for understanding the chemical history and evolution of clusters. A large amount of metals in the ICM are mainly produced by supernovae ( $\mathrm{SNe}$ ) in galaxies, and are classified roughly as Type Ia (SNe Ia) and Type II (SNe II). Elements such as $\mathrm{Si}, \mathrm{S}$ and $\mathrm{Fe}$ are synthesized in both SNe Ia and SNe II, while lighter $\alpha$ elements such as $\mathrm{O}, \mathrm{Ne}$, and $\mathrm{Mg}$ are mainly produced in SN II, which are explosions of massive stars with initial mass above $\sim 10 M_{\odot}$. The metals produced in the galaxies are transferred into the ICM by galactic wind and/or ram pressure strippings.

Recent observational studies of clusters with Chandra and XMM-Newton, with their powerful imaging capability and large effective area, have unveiled radial profiles of temperature, entropy, gas mass, and total mass of the ICM up to $r_{500}$ (e.g., [25, 37, 39]). The derived temperature and entropy profiles in the outer region to $r_{500}$ were almost consistent with theoretical expectations from the self-similar assumption. Also, Zhang et al. [39] showed that the gas mass fraction $M_{\text {gas }} / M_{\text {total mass }}$ increases with radius to $r_{500}$.

As for metal abundances, ASCA first measured the distributions of Si and Fe in the ICM (e.g., [8]). The derived iron-mass to light ratios (IMLR) are nearly constant in rich clusters and decrease toward poorer systems [19. Recent observations with Chandra and XMM-Newton allowed detailed studies of the metals in the ICM. These observations, however, showed abundance profiles of $\mathrm{O}, \mathrm{Mg}, \mathrm{Si}$ and $\mathrm{Fe}$ only for the central regions of very bright clusters or groups of galaxies dominated by $\mathrm{cD}$ galaxies in a reliable manner (e.g., [7, 9, 20, 35]). The abundance profiles of $\mathrm{O}$ and $\mathrm{Mg}$, in particular for outer regions of clusters, are still poorly determined, because data from Chandra and XMM-Newton both show relatively high intrinsic background levels. Tamura et al. [35] derived IMLR for five clusters within $250 h_{100}^{-1} \mathrm{kpc}$ to be $\sim 0.01 M_{\odot} / L_{\odot}$, and the oxygen mass within $50 h_{100}^{-1} \mathrm{kpc}$ for several clusters. However, oxygen-mass to light ratios (OMLR) for rich clusters are not reliable due to the low emissivity of 
$\mathrm{O}_{\mathrm{VII}}$ and $\mathrm{O}_{\mathrm{VIII}}$ lines in high temperatures. De Grandi et al. [5, 6] and Hayakawa et al. [13] found that clusters associated with $\mathrm{cD}$ galaxies and central cool components showed abundance concentration in the cluster center, while clusters without $\mathrm{cD}$ galaxies indicated flatter profiles. The central metallicity enhancement in the cool core clusters were further studied and the excess metals were shown to be supplied from the $\mathrm{cD}$ galaxies [6].

The spatial distribution and elemental abundance pattern of the ICM metals were determined with the large effective area of XMM-Newton (e.g., [21, 22]). On the other hand, abundance measurements of $\mathrm{O}$ and $\mathrm{Mg}$ with XMM-Newton could only be made for the central regions of the brightest cooling core clusters, due to the relatively high intrinsic background. Rasmussen et al. [26, 27] report the Si and Fe profiles of 15 groups of galaxies observed with Chandra. They suggest that the $\mathrm{Si}$ to $\mathrm{Fe}$ ratios in the groups tend to increase with radius, and the IMLRs within $r_{500}$ show a positive correlation with the total group mass (temperature).

We use $H_{0}=70 \mathrm{~km} \mathrm{~s}^{-1} \mathrm{Mpc}^{-1}, \Omega_{\Lambda}=1-\Omega_{\mathrm{M}}=$ 0.73 in this paper. Unless otherwise noted, the solar abundance table is given by Anders \& Grevesse [2], and errors are within the $90 \%$ confidence region for a single parameter of interest.

\section{Thermal PROperties OF THE ICM}

\subsection{Temperature Profiles}

Because Suzaku XIS is characterized by a lower background level and higher sensitivity below $1 \mathrm{keV}$ [18], we have been able to observe the ICM emission beyond $r_{500}$ region of clusters [1, 4, 10, 12, 15, 28, 34]. Almost all clusters observed with Suzaku showed a similar trend, which the temperature dropping to $\sim 1 / 3$ of the peak value. This was consistent with the theoretical expectations as shown in Fig. 1. The temperature was normalized by the mean temperatures of the clusters. The radial axis in Fig. 1 was normalized by $r_{200}$, which was derived from the mean temperature of the clusters in Henry et al. 11. Dotted line show simulation result (Burns et al. 2010), and two gray dashed lines show standard deviation.

\subsection{ENTROPY PROFILES}

An entropy profile provides the thermal process and history of the ICM, particularly for the gas heated by the accretion shock from outside the cluster. In X-ray astronomy, we define the entropy as $S=k T n_{\mathrm{e}}^{-2 / 3}$. The derived entropy profile from Suzaku increased with the radius to $\sim 0.5 r_{200} \sim r_{500}$, and the profile had a flatter slope at $r>0.5 r_{200}$. This tendency was consistent for the Suzaku results [1, 4, 10, 12]. Compared to the previous XMM results for 31 clusters within $r_{500}$ in Pratt et al. [25], Suzaku's results were consistent with the entropy profile within $r_{500}$.

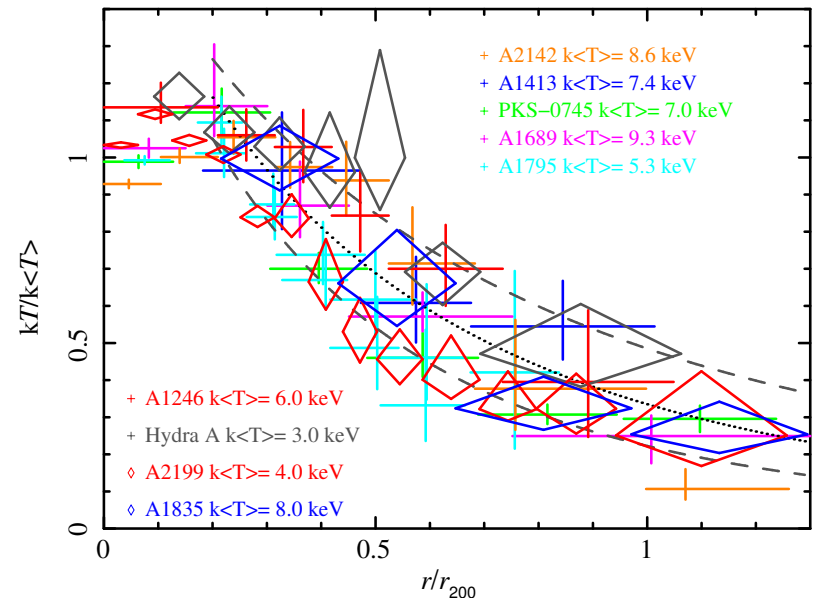

FigURE 1. Radial scaled temperature profiles by the mean temperature for the clusters observed with Suzaku (see also [1]). The radii are normalized by $r_{200}$ with the mean temperature as shown in Henry et al. [11.

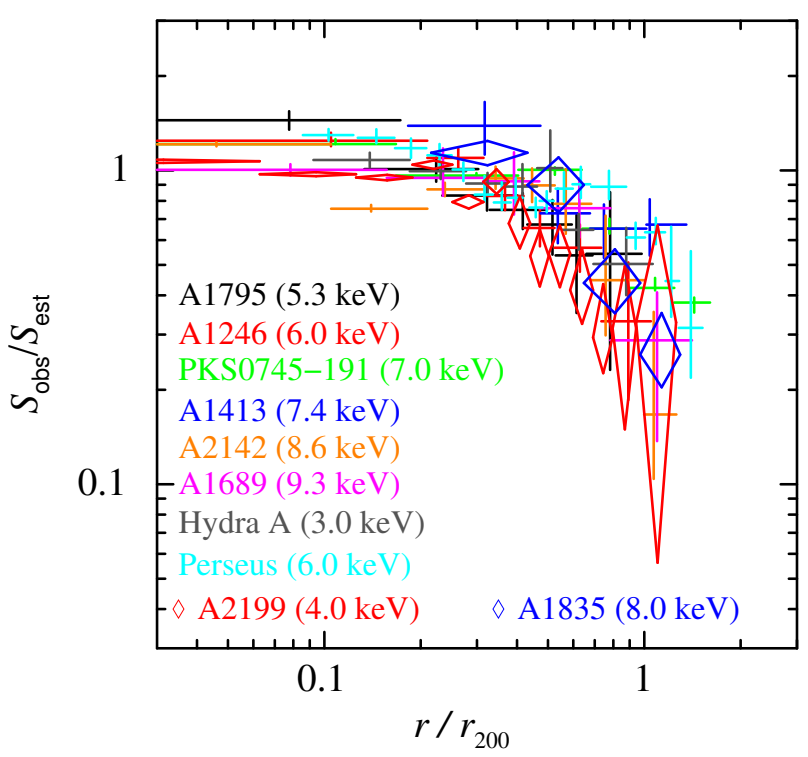

FiguRE 2. Derived radial entropy profile for each cluster from Suzaku observations. The radii are normalized by $r_{200}$ with the mean temperature as shown in Henry et al. [11.

Voit [38] reported $S \propto r^{1.1}$ on the basis of numerical simulations of adiabatic cool gas accretion and the XMM results. Pratt et al. 25] agreed with the relation within $r_{500}$. We compared the entropy derived from Suzaku with the expected values from simulations, as shown in Fig. 2 As a result, all the observed clusters had a similar tendency in $r>0.2 r_{200}$. This would suggest that all the observed clusters have undergone a similar thermal evolution process.

\subsection{MASS PROFILE}

While the gas mass of the clusters is calculated by the electron density from X-ray observation, the gravitational mass of the clusters is derived from the temperature and electron density profiles assuming spherical 


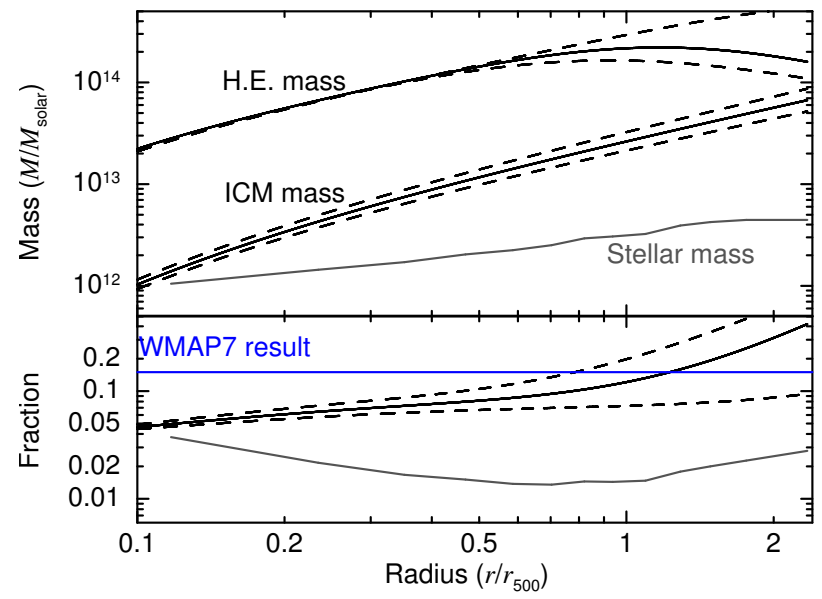

Figure 3. Total mass under the assumption of hydrostatic equilibrium and gas mass (upper and lower solid lines, respectively) of the Abell 2199 cluster. Each dashed line shows $\pm 90 \%$ errors for each mass. The gray line corresponds to the stellar mass from K-band luminosity with the 2MASS catalogue. Lower panel: The radial profile of the stellar and gas mass fraction to the total cluster mass. The dashed lines show $\pm 90 \%$ errors. The blue line indicates the cosmic baryon fraction [16].

symmetry and hydrostatic equilibrium. For example, the stellar, gas and total mass of the Abell 2199 cluster are shown in Fig. 3. Note that the resultant total mass from the hydrostatic equilibrium assumption started decreasing beyond the $r_{500}$ region. This would indicate a flaw in the assumption. As mentioned in the previous section, the flatness or a decrease of the entropy in $r>r_{500}$ would also indicate being outside the hydrostatic equilibrium assumption in the outskirts region of the cluster. The fraction of the derived baryon to the total mass of the cluster at $r_{200}$ was $\sim 20 \%$, which was consistent with the cosmic baryon fraction, $\sim 15 \%$ [16].

\section{Metal distributions in the ICM}

\subsection{Metallicity IN THE ICM}

Suzaku XIS can measure all the main elements from O to Fe, because it realizes a lower background level and higher spectral sensitivity, especially below $1 \mathrm{keV}$ [18]. Suzaku observations have shown the abundance profiles of $\mathrm{O}, \mathrm{Mg}, \mathrm{Si}, \mathrm{S}$, and $\mathrm{Fe}$ to the outer regions $\left(r \sim 0.5 r_{180}\right)$ with good precision for several groups and clusters [17, 21, 22, 29, 34, 36]. Because the Fe abundance was well determined in the metal abundances with smaller uncertainties, we compared the distributions of the metal abundances with those of the Fe abundance. In order to compare the relative variation in the abundance profiles, we show the abundance ratios of $\mathrm{O}, \mathrm{Mg}, \mathrm{Si}$, and $\mathrm{S}$ divided by $\mathrm{Fe}$ as a function of the projected radius in Figs. 47. As a result, the $\mathrm{Si}$ and $\mathrm{S}$ to $\mathrm{Fe}$ abundance ratios were close to $\sim 1.5$ from the central to the outer region, while the gradients of the $\mathrm{O}$ and $\mathrm{Mg}$ to $\mathrm{Fe}$ ratios increase

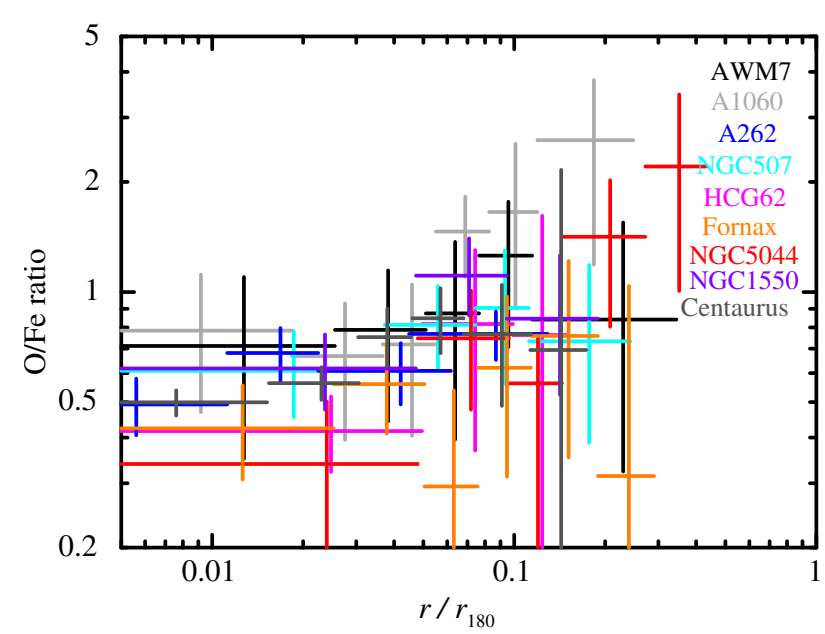

Figure 4. Radial abundance ratios of $\mathrm{O}$ to $\mathrm{Fe}$.

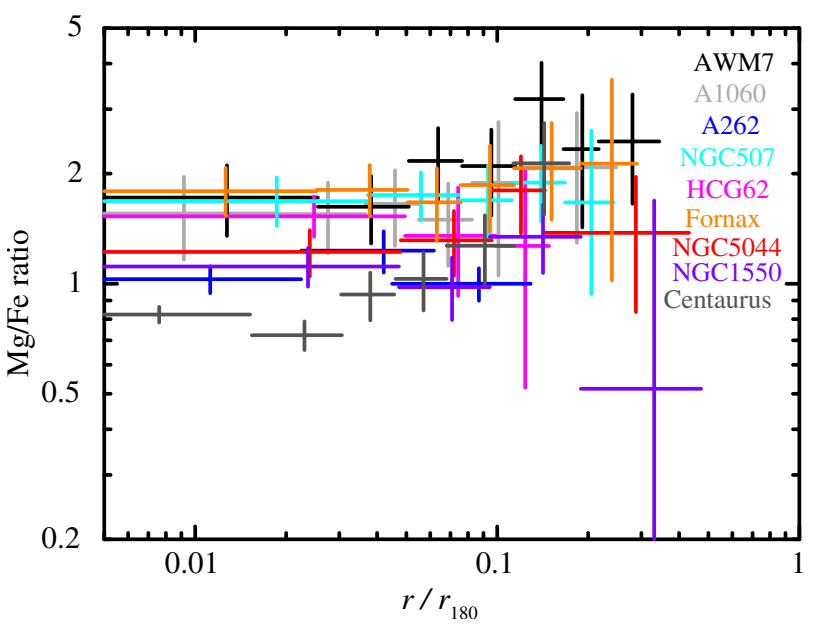

Figure 5. Radial abundance ratios of $\mathrm{Mg}$ to $\mathrm{Fe}$.

more gently than those of the $\mathrm{Si}$ and $\mathrm{S}$ to Fe ratios, as shown in Figs. 4 7. In addition, the O/Fe solar abundance ratios in the central region were about $\sim 0.6$, while the other elements to Fe ratios in the central region were almost a solar abundance. The feature of $\mathrm{O}$ abundance agreed with the XMM-Newton observations [21, 22, 35].

\subsection{CONTRIBUtions FROM TYPE IA AND TYPE II SUPERNOVAE}

In order to examine the relative contributions from type Ia and type II supernovae (SNe Ia and SNe II) to the ICM metals, the elemental mass pattern of $\mathrm{O}, \mathrm{Mg}, \mathrm{Si}, \mathrm{S}$ and $\mathrm{Fe}$ was examined for each cluster. The mass patterns were fitted by a combination of average SNe Ia and SNe II yields per supernova, as demonstrated in Fig. 8. The fit parameters were chosen to be the integrated number of SNe Ia $\left(N_{\text {Ia }}\right)$ and the number ratio of SNe II to SNe Ia $\left(N_{\text {II }} / N_{\text {Ia }}\right)$, because $N_{\text {Ia }}$ could be well constrained due to relatively small errors in the Fe abundance. The SNe Ia and II yields were taken from Iwamoto et al. 14 and Nomoto et al. 24, respectively. We assumed a Salpeter IMF for stellar masses from 10 to $50 M_{\odot}$ with progenitor 


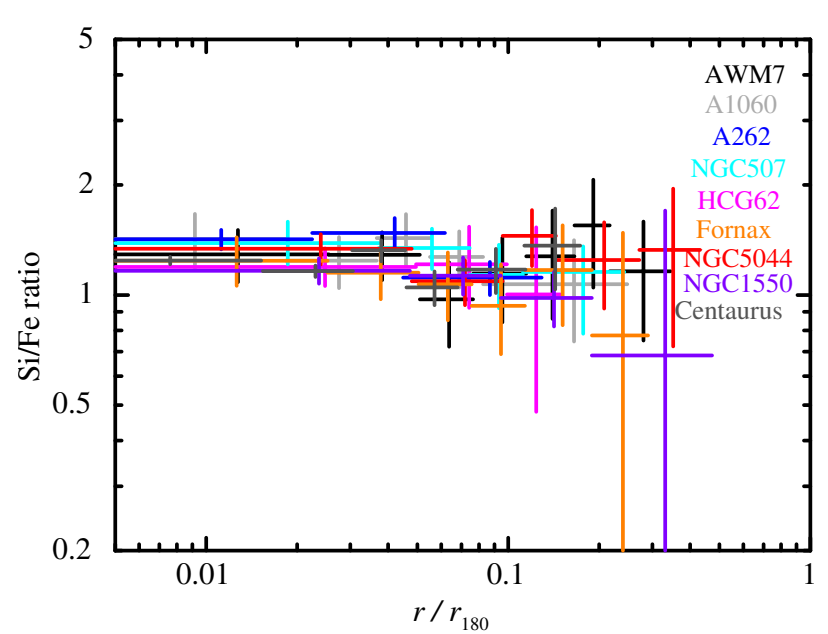

Figure 6. Radial abundance ratios of Si to Fe.

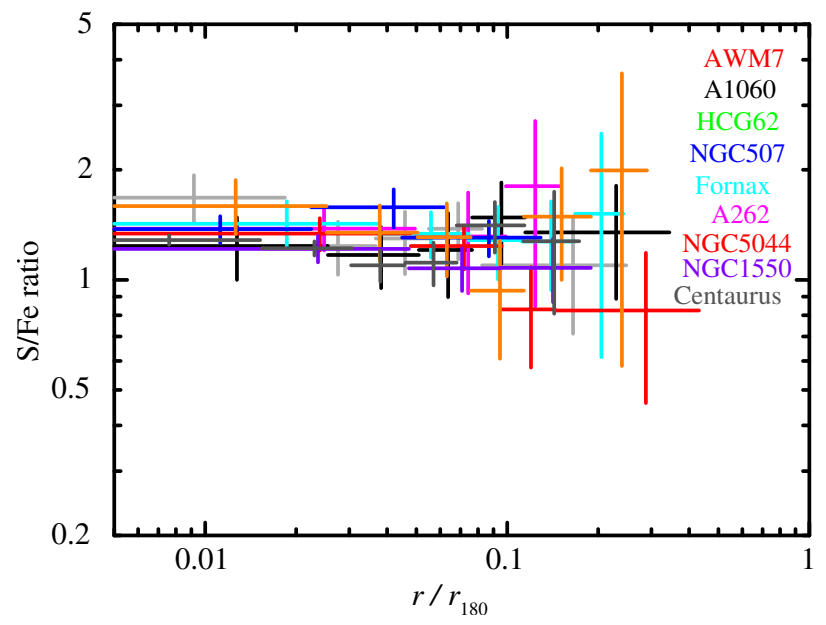

Figure 7. Radial abundance ratios of $\mathrm{S}$ to Fe.

metallicity of $Z=0.02$ for SNe II, and W7, WDD1 or WDD2 models for SNe Ia. All the clusters exhibited similar features. The abundance patterns were better represented by the W7 SNe Ia yield model than by WDD1. The number ratio of SNe II to SNe Ia with $\mathrm{W} 7$ is $\sim 3.5$, while the ratio with WDD1 is $\sim 2.5$. The WDD2 model gave quite similar results as W7. Almost $3 / 4$ of the $\mathrm{Fe}$ and $\sim 1 / 4$ of the $\mathrm{Si}$ is synthesized by $\mathrm{SNe}$ Ia, in the W7 model (for details, see Sato et al. [29, 30]). Note that, here, we estimated the numbers as the integrated ones at present. We would therefore need to consider the instantaneous recycling approximations, taking into account the stellar lifetime etc., as shown in Matteucci \& Chiappini [23], to derive the correct chemical evolution of the galaxies in the clusters.

\subsection{Metal mass to Light RAtios}

We examined mass-to-light ratios for $\mathrm{O}, \mathrm{Fe}$, and $\mathrm{Mg}$ (OMLR, IMLR, and MMLR, respectively), which enabled us to compare the ICM metal distribution with the stellar mass profile. Historically, B-band luminosity has been used for estimating the stellar mass [19]. However we calculated it using the K-band luminosity in the clusters, based on the Two Micron All Sky

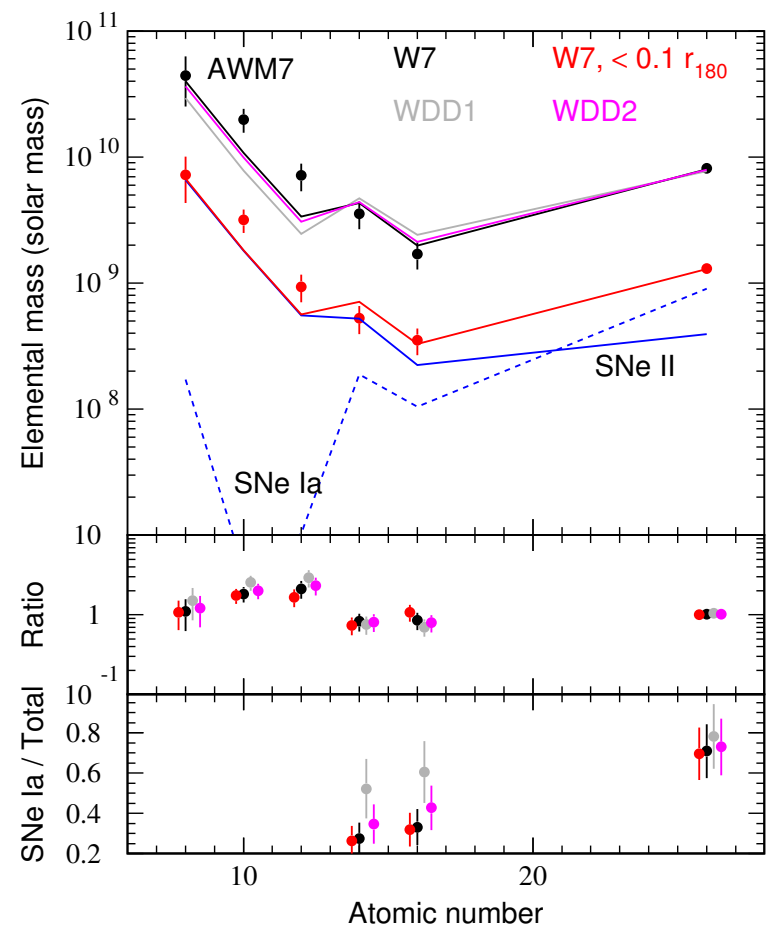

Figure 8. Fit results of each elemental mass for the AWM 7 cluster. Top panels show the mass within the whole observed region (black) and within $0.1 r_{180}$ (red) fit by $\left[N_{\text {Ia }}\left\{(\right.\right.$ SNe Ia yield $)+\left(N_{\text {II }} / N_{\text {Ia }}\right)($ SNe II yield $\left.\left.)\right\}\right]$. The blue dashed and solid lines correspond to the contributions of SNe Ia (W7) and SNe II within $0.1 r_{180}$, respectively. Ne (atomic number $=10$ ) is excluded in the fit. The mid and lower panels indicate the ratios of data points to the best-fit, and the fractions of the SNe Ia contribution to the total mass in the best-fit model for each element, respectively.

Survey (2MASS) catalogue. This method is useful in performing a uniform comparison with the properties in other groups and clusters based on the same K-band galaxy catalogue to trace the distribution of member elliptical galaxies. The MLRs with K-band continue to increase in the observed region with Suzaku, as shown in Figs. 9 11. This means that the metals extend to the outer region than the galaxies. The MLRs with K-band also exhibited a similar tendency for each cluster, and showed a smaller dispersion than those with B-band luminosity. These suggest that the metal enrichment process in the ICM seems to be similar for each cluster.

We stress that high-sensitivity abundance observation to the outer region of clusters will give important clues about their evolution. If the distribution of oxygen, as well as iron, could be measured to the very outer region $\left(r \sim r_{180}\right)$, we might obtain a clear view about when the oxygen and iron were supplied to the inter galactic space, because most of the oxygen should have been synthesized by SNe II and supplied in the starburst era. Another possibility is very early metal enrichment of oxygen by galaxies or by massive Population III stars before groups and clusters assem- 


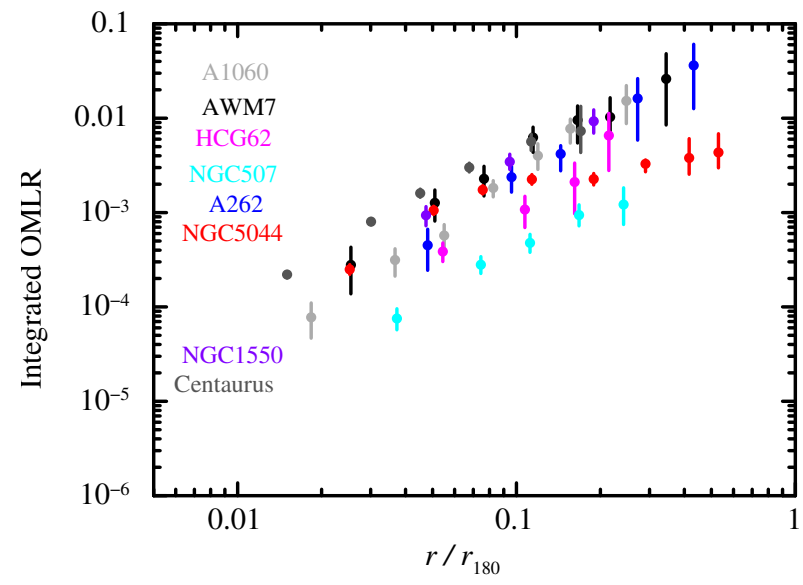

Figure 9. Radial oxygen mass-to-light profiles with K-band luminosity.

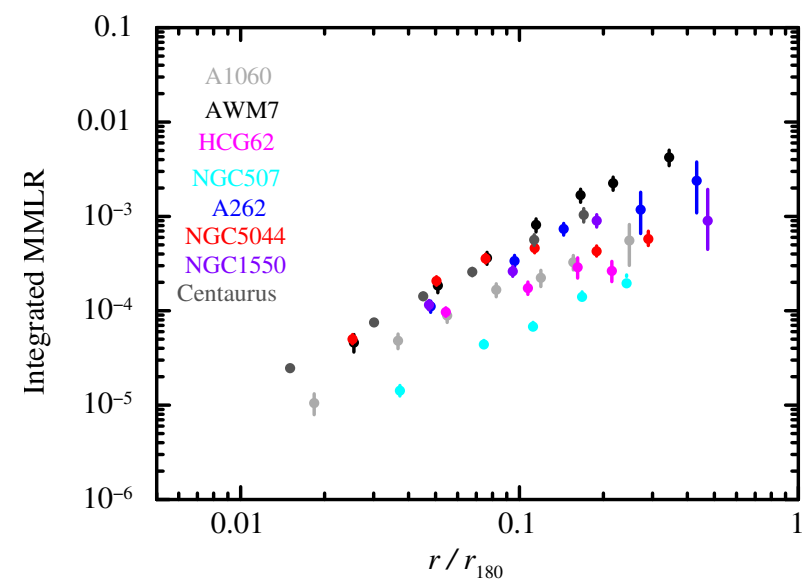

FigURE 10. Radial magnesium mass-to-light profiles with K-band luminosity.

bled. In this case, a large part of the intergalactic space would be enriched quite uniformly with oxygen and other elements. Metallicity information in cluster outskirts would thus give us unique information about the enrichment history. For this purpose, instruments with much higher energy resolution, such as microcalorimeters, and optics with larger effective area will play a key role in carrying out these studies.

\section{Conclusion}

Suzaku observations of group and cluster galaxies for the first time showed spatial distributions of temperature and entropy to the virial radii, and metal abundances for $\mathrm{O}, \mathrm{Mg}, \mathrm{Si}, \mathrm{S}$, and Fe up to $\sim 0.5 r_{180}$. The ICM temperatures at the virial radii dropped by $\sim 30 \%$ from the peak temperature. The derived entropy profiles from Suzaku were consistent with those from the previous XMM results and the expected value from numerical simulations within $r<r_{500}$. In $r>r_{500}$, the derived entropy profiles had a flatter slope than that expected from the simulations. This would indicate that the outskirts of the cluster were outside the hydrostatic equilibrium assumption.

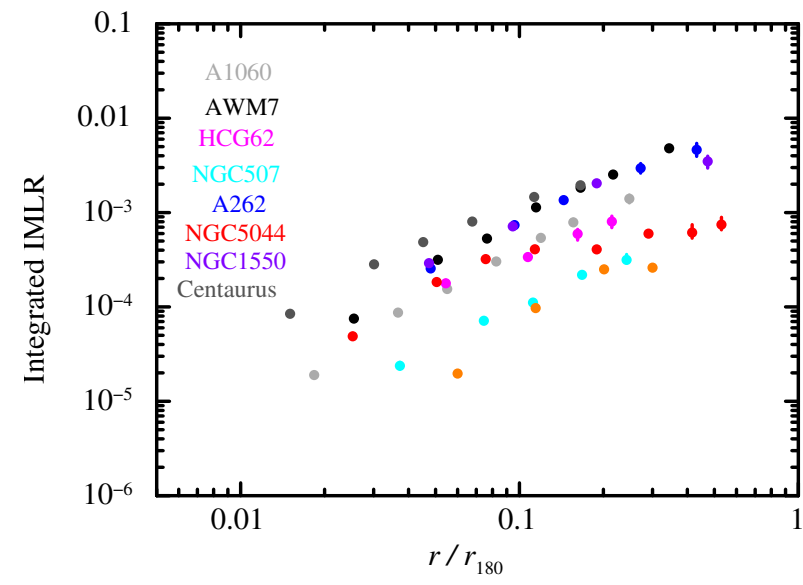

FiguRE 11. Radial iron mass-to-light profiles with K-band luminosity.

The abundances of $\mathrm{Mg}, \mathrm{Si}, \mathrm{S}$, and Fe dropped from solar levels at the center to $\sim 1 / 4$ solar in the outermost region, while the $\mathrm{O}$ abundance showed a flatter distribution around $\sim 0.5$ solar without a strong concentration in the center. The abundance ratios, $\mathrm{O} / \mathrm{Fe}$, $\mathrm{Mg} / \mathrm{Fe}, \mathrm{Si} / \mathrm{Fe}$, and $\mathrm{S} / \mathrm{Fe}$ in the groups and clusters were generally similar to each other. The abundance pattern from $\mathrm{O}$ to $\mathrm{Fe}$ enabled us to constrain the number ratio of SNe II to Ia as $\sim 3.5$, which was consistent with the values obtained for the groups and clusters. The derived OMLR, MMLR, and IMLR in the clusters using K-band luminosity had a similar feature. This would suggest that the clusters had undergone a similar evolution process in the past.

\section{REFERENCES}

[1] Akamatsu, H., Hoshino, A., Ishisaki, Y., Ohashi, T., Sato, K., Takei, Y., \& Ota, N. 2011, arXiv:1106.5653

[2] Anders, E., \& Grevesse, N. 1989, Geochim.

Cosmochim. Acta, 53, 197

[3] Barnes, J., \& Efstathiou, G. 1987, ApJ, 319, 575

[4] Bautz, M. W., et al. 2009, PASJ, 61, 1117

[5] De Grandi, S., \& Molendi, S., 2001 ApJ, 551, 153

[6] De Grandi, S., Ettori, S., Longhetti, M., \& Molendi, S. 2004, A\&A, 419, 7

[7] Finoguenov, A., Matsushita, K., Böhringer, H., Ikebe, Y., \& Arnaud, M. 2002, A\&A, 381, 21

[8] Fukazawa, Y., Makishima, K., Tamura, T., Ezawa, H., Xu, H., Ikebe, Y., Kikuchi, K., \& Ohashi, T. 1998, PASJ, 50, 187

[9] Fukazawa, Y., Makishima, K., \& Ohashi, T. 2004, PASJ, 56, 965

[10] George, M. R., Fabian, A. C., Sanders, J. S., Young, A. J., \& Russell, H. R. 2009, MNRAS, 395, 657

[11] Henry, J. P., Evrard, A. E., Hoekstra, H., Babul, A., \& Mahdavi, A. 2009, ApJ, 691, 1307

[12] Hoshino, A., et al. 2010, PASJ, 62, 371

[13] Hayakawa, A., Hoshino, A., Ishida, M., Furusho, T., Yamasaki, N. Y., \& Ohashi, T. 2006, PASJ, 58, 743 
[14] Iwamoto, K., Brachwitz, F., Nomoto, K., Kishimoto, N., Umeda, H., Hix, W. R., \& Thielemann, F.-K. 1999, ApJS, 125, 439

[15] Kawaharada, M., et al. 2010, ApJ, 714, 423

[16] Komatsu, E., et al. 2011, ApJS, 192, 18

[17] Komiyama, M., Sato, K., Nagino, R., Ohashi, T., \& Matsushita, K. 2009, PASJ, 61, 337

[18] Koyama, K., et al. 2007, PASJ, 59, 23

[19] Makishima, K., et al. 2001, PASJ, 53, 401

[20] Matsushita, K., Finoguenov, A., Böhringer, H. 2003, A\&A, 401, 443

[21] Matsushita, K., Böhringer, H., Takahashi, I., \& Ikebe, Y. 2007b, A\&A, 462, 953

[22] Matsushita, K., et al. 2007a, PASJ, 59, 327

[23] Matteucci, F., \& Chiappini, C. 2005, PASA, 22, 49

[24] Nomoto, K., Tominaga, N., Umeda, H., Kobayashi, C., \& Maeda, K. 2006, Nuclear Physics A, 777, 424

[25] Pratt, G. W., et al. 2010, A\&A, 511, A85

[26] Rasmussen, J., \& Ponman, T. J. 2007, MNRAS, 380, 1554

[27] Rasmussen, J., \& Ponman, T. J. 2009, MNRAS, 399, 239
[28] Reiprich, T. H., et al. 2009, A\&A, 501, 899

[29] Sato, K., et al. 2007, PASJ, 59, 299

[30] Sato, K., Tokoi, K., Matsushita, K., Ishisaki, Y., Yamasaki, N. Y., Ishida, M., \& Ohashi, T. 2007, ApJ, 667, L41

[31] Sato, K., Matsushita, K., Ishisaki, Y., Yamasaki, N. Y., Ishida, M., Sasaki, S., \& Ohashi, T. 2008, PASJ, $60, \mathrm{~S} 333$

[32] Sato, K., Matsushita, K., Ishisaki, Y., Yamasaki, N. Y., Ishida, M., \& Ohashi, T. 2009a, PASJ, 61, 353

[33] Sato, K., Matsushita, K., \& Gastaldello, F. 2009b, PASJ, 61, 365

[34] Sato, K., Kelley, R. L., Takei, Y., Tamura, T., Yamasaki, N. Y., Ohashi, T., Gupta, A., \& Galeazzi, M. 2010, PASJ, 62, 1423

[35] Tamura, T., Kaastra, J. S., Makishima, K., \& Takahashi, I. 2003, A\&A, 399, 497

[36] Tokoi, K., et al. 2008, PASJ, 60, S317

[37] Vikhlinin, A., Markevitch, M., Murray, S. S., et al. 2005, ApJ, 628, 655

[38] Voit, G. M. 2005, Reviews of Modern Physics, 77, 207

[39] Zhang, Y.-Y., Okabe, N., Finoguenov, A., et al. 2010, ApJ, 711, 